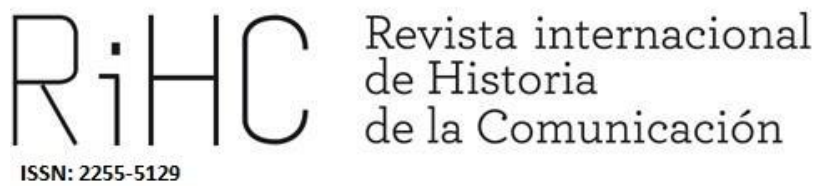

\title{
LA PRENSA Y EL PERIODISMO EN LA REVOLUCIÓN PORTUGUESA (1974-1975)
}

Press and journalism in Portuguese Revolution (1974-1975)

DOI: https://dx.doi.org/10.12795/RiHC.2020.i14.12

Recibido: $17 / 02 / 2020$

Aceptado: $16 / 04 / 2020$

Publicado: $15 / 06 / 2020$

Pedro Marques Gomes

ORCID (iD) 0000-0002-3189-3388

IHC - Universidade Nova de Lisboa

ESCS - Instituto Politécnico de Lisboa

pedrogomes@fcsh.unl.pt 
Resumen: Los órganos de comunicación social jugaron un papel muy relevante durante el periodo revolucionario (1974-1975) que siguió a la caída de la dictadura portuguesa, actuando no solo como vehículos de transmisión de información a los ciudadanos, sino también como actores participantes en el proceso político.

En este artículo, analizamos la prensa portuguesa durante esos años determinantes, procurando destacar sus principales características y las transformaciones que sufrió. Se observa la aparición de un tipo de periodismo "revolucionario" o "militante", en una coyuntura caracterizada por grandes combates políticos, pues estaba en juego la definición del tipo de régimen que habría de implantarse en Portugal.

Palabras clave: Prensa; Periodismo; Revolución de Abril; Luchas políticas; Portugal.

Abstract: The media had a relevant role in the revolutionary period (1974-1975) after the overthrow of the $p$

Portuguese dictatorship, not only as vehicles of transmitting information to citizens, but also as actors in the political process.

In this article, we analyse the Portuguese press from the context of revolution and identify its mains characteristics and transformations. We observe the emergence of the type of "revolutionary" or "militant" journalism, at a time of great political fights, when discussing the definition of type of regime to be implemented in Portugal.

Keywords: Press; Journalism; April revolution; Political fights; Portugal.

\section{Introducción}

Antes del golpe militar del 25 de abril de 1974, la mayoría de los periódicos portugueses pertenecían a grandes grupos económicos. Se vivió una fuerte censura y, aunque, hemos visto muchos cambios en la prensa, con algunas publicaciones periódicas haciendo la oposición "posible" a la dictadura, la verdad es que el alcance de la acción de los periodistas anteriores era muy pequeño.

Con el 25 de abril, la abolición de la censura y la posibilidad de construir un país democrático, se han producido muchos cambios en el sector de los medios de comunicación portugueses. Sin embargo, también han surgido varios proyectos de ley que pretendían limitar la libertad de prensa, con diferentes justificaciones, pero sobre todo, argumentando que la publicación de algunos contenidos podría causar aún más inestabilidad en la sociedad portuguesa (Mesquita, 1988, 1994).

La libertad de prensa y el control de los medios, siendo fundamentales para la definición de los regímenes políticos, estuvieron, desde el principio, presentes en los enfrentamientos que existieron entre las diferentes corrientes de la sociedad portuguesa. Los periódicos estuvieron involucrados y participaron activamente en el curso de los eventos, la agitación revolucionaria se vivió también en las salas de 
redacción y la forma de practicar el periodismo en este momento seria marcada por este descontento.

En este artículo, buscamos comprender cómo los periódicos y periodistas vivieron el proceso revolucionario (1974-1975), tratando de resaltar las principales tendencias y características de la forma de practicar el periodismo, el medio ambiente en las salas de redacción, así como, las relaciones complejas entre la política y el periodismo. Para este estudio, utilizamos la bibliografía existente sobre los medios en la revolución, y también los artículos de prensa y los testimonios de periodistas de la época, algunos que fueron publicados en libros, otros que fueron recopilados a través de entrevistas orales, donde intentamos recuperar los recuerdos de esa época, en primera persona y según la experiencia de profesionales de diferentes periódicos.

El artículo está estructurado en cinco partes fundamentales. Después de una breve introducción, se presenta una síntesis del estado del arte en los medios de comunicación en la revolución portuguesa. Enseguida, se presenta una narración cronológica donde se aborda la prensa del día 25 de abril y en otros dos períodos fundamentales de la revolución, revelando sus especificidades con respecto a la prensa: hasta el 11 de marzo de 1975 y desde entonces hasta el 25 de abril. Se termina con notas finales.

\section{Estado de la cuestión}

Entre las múltiples transformaciones que, a partir del 25 de Abril de 1974, experimenta la sociedad portuguesa, destacan las que afectan al sector de la comunicación social, que cambiará de forma radical, como coinciden en afirmar las diferentes obras académicas que se han ocupado sobre el tema.

Precursor en la investigación sobre la evolución de los medios de comunicación portugueses durante el periodo revolucionario (1974-1975), para Mário Mesquita, tras la Revolución de los Claveles, "los periódicos estaban afinados por el mismo diapasón: la glorificación de los vencedores militares y civiles" (Mesquita, 1994b: 98). En una línea semejante, Francisco Rui Cádima señala que, en esos primeros meses, "los media, de alguna manera, reflejaban un cierto servilismo político al MFA" y, por eso, "apelaban constantemente a la necesidad de que el MFA protegiese a la comunicación social de los discursos de la "reacción»" (Cádima, 2001: 328). Si, en esta primera fase, el discurso periodístico se identificaba con los planteamientos de los militares del MFA, la propia evolución del proceso revolucionario haría revelar otras tendencias. Así, como observa João Figueira, pasa a practicarse "un periodismo cargado de ideología, en que la política es una urgencia, tras 48 años de dictadura y ausencia de libertad de expresión y de pensamiento" (Figueira, 2007: 21). 
La prensa escrita acompañaba el curso de la revolución, reflejando las nuevas alternativas y orientaciones que fueron surgiendo, bien por la división del MFA en varias facciones, bien por el protagonismo que a lo largo del periodo fueron ganando los partidos políticos, así como por los cambios de los principales temas que eran objeto de debate en la sociedad portuguesa, entre otros factores. De esta forma, si - como sugiere Mário Mesquita - antes del 28 de septiembre de 1974 "eran raros los periódicos que habían informado sobre las divergencias [que existían] entre la Junta de Salvación Nacional y la "coordinadora» del MFA, entre Spínola y Costa Gomes, entre el MFA de los capitanes y el MFA de los generales" (Mesquita, 1988: 101), en pleno "Verano Caliente» de 1975 -como apunta el mismo autor en un artículo posterior- la "prensa gonçalvista fue subiendo de tono" y la "agresividad se conjugaba con la arrogancia" (Mesquita, 1994b: 382; 362).

El análisis discursivo llevado a cabo por João Figueira (2007) a tres de las principales cabeceras de Lisboa muestra cómo estos periódicos interpretaban de manera diferente los mismos acontecimientos y la acción de los protagonistas del proceso revolucionario, defendiendo implícitamente distintas orientaciones para el futuro del país. En la prensa de Oporto, de acuerdo con la investigación realizada por Helena Lima, también eran notorias las discrepancias en la orientación de los periódicos, reflejándose, en líneas generales, las principales tendencias que atravesaban la prensa nacional:

El Comércio do Porto se consolidó como el título que mantenía la batalla contra la deriva izquierdista de la sociedad portuguesa, mientras el Jornal de Notícias era denominado peyorativamente Pravda, porque se caracterizaba por un tono muy radicalizado. En cuanto a $O$ Primeiro de Janeiro, por más que su línea editorial no siguiera una orientación muy determinada, su redacción también pasó por una situación de pulverización de autoridad (Lima, 2008: 350).

Se trata de cuatro autores insoslayables en los estudios sobre los media y la revolución, y cuyos trabajos son, en cierto sentido, complementarios. Mesquita (1988; 1994b; 2019) presenta una visión estructurada de lo que era el sistema mediático portugués en aquellos años, interpretando estrategias de políticos, militares y periodistas, poniéndolas en contexto y dando razón de algunas variables fundamentales para la comprensión de la acción de los órganos de comunicación social durante aquel periodo: la legislación producida, los cambios en los contenidos que se publicaban en la prensa, los principales casos que afectaron a los medios de comunicación, las características de los periodistas, el papel del Estado, etc. Por su parte, aunque también se ocupe de algunas de estas cuestiones, Rui Cádima (2001) se centra en los artículos que fueron saliendo en la prensa, lo que le permite destacar cambios en los enfoques y los temas.

Por su parte, João Figueira y Helena Lima realizaron sus investigaciones en el contexto de sus estudios de máster y doctorado, respectivamente. El primero, tras definir un corpus de análisis formado por los periódicos Expresso, Diário de Notícias y Jornal Novo, 
dentro de un ámbito cronológico restringido al "Verano Caliente» de 1975, analiza críticamente el discurso entonces producido. La segunda optará por el estudio de los periódicos en sí mismos, buscando entender lo que cambió y lo que perduró en la prensa de la ciudad de Oporto. Además del estudio del periodo revolucionario, la autora analiza también los mismos títulos en momentos posteriores.

Desde ámbitos diversos, en los últimos años se han publicado algunos otros trabajos, que contribuyen a una mejor comprensión del tema, al tiempo que permiten observarlo bajo diferentes ángulos. En este contexto destacan investigaciones que abordan casos particulares, como los de los periódicos República y Diário de Notícias y también el de la cadena radiofónica Rádio Renascença (Mesquita, 1994a; Ribeiro, 2002; Gomes, 2014; Paulo, 2014), los cambios que se produjeron en la Emisora Nacional y en la televisión pública (RTP) (Rezola, 2014 y 2019; Santos, 2017; Baptista, 2019), las agencias noticiosas (Fonseca y Carvalho, 2016), la prensa partidaria (Sousa, 2003), o la aparición de nuevos periódicos privados (Gomes, 2018).

\section{La prensa y el 25 de abril de 1974}

Los órganos de comunicación social portugueses tuvieron un papel determinante en las operaciones del 25 de Abril de 1974, así como en los días inmediatamente siguientes. Desde la transmisión de las contraseñas revolucionarias por la radio, a la presentación de la Junta de Salvación Nacional (JSN) 1 en la televisión, pasando por la divulgación de los primeros comunicados y la publicación del Programa del Movimiento de las Fuerzas Armadas (MFA), hasta la difusión a través de la prensa de las tomas de posición de políticos y militares, son varios los ejemplos que pueden citarse de esa relevancia.

No sorprende, por eso, la agitación que se vive en las redacciones a partir de la madrugada del día 25. El ambiente era también de duda, esencialmente por las pocas informaciones que llegaban, algunas de las cuales contradictorias. Dentro y fuera de los periódicos, se buscaban fuentes que permitieran explicar lo que estaba sucediendo en las calles de Lisboa. En el Diário de Notícias (DN), que era considerado el diario "oficioso" del régimen, la periodista Manuela de Azevedo relata esas horas de alborozo. "Las noticias se multiplicaban desconcertantes. El director del periódico ingenuamente insistía en que los textos debían remitirse a la Censura, pero Santos Jorge [subjefe de redacción] le contradecía: - Ya no hay Censura" (Azevedo 2010, 207).

1 Compuesta exclusivamente por militares, la JSN ejerció el poder de forma interina hasta la formación del primer gobierno provisional. 
El hecho de que este periódico ha sido fuertemente vinculado al régimen depuesto con trabajadores ideológicamente cercanos y otros con un camino de oposición a la dictadura, dará lugar a un clima de tensión dentro de él. A pesar de esto, como dice José António Santos, reinaba la alegría al final de la dictadura: "No puedo ocultar que en algunos rostros vi sorpresa y desánimo, porque había compañeros en nuestra profesión que estaban alienados con el Estado Novo y se dieron cuenta de que el sueño se había ido. Pero, el gran sentimiento en la sala de redacción es de alegría y satisfacción" (José António Santos en entrevista con el autor, Lisboa, 2012). En efecto, gran parte de los periódicos ya no somete los textos al análisis de los censores.

Pero, más allá de la decisión de enviar o no las pruebas al Examen Previo, había que informar sobre los acontecimientos que estaban ocurriendo en la capital. En el República, Eugénio Alves y José Jorge Letria firmaron el reportaje del día:

Cerca de las 7:30 nos dirigimos a la redacción del República, agotados por una noche en vela inolvidable y por la tensión nerviosa provocada por la incertidumbre y por la espera, y escribimos el primer reportaje de aquella madrugada histórica, que saldría en una edición ya liberada de la intervención de la censura. Fue la primera de las varias que llegaron a la calle ese día, anunciando que el Movimiento de las Fuerzas Armadas había tomado el poder en Lisboa y en otros puntos del país (Letria, 2013: 158).

También hubo periodistas vinculados al ejército de la MFA, que por lo tanto tenían información privilegiada sobre las operaciones. Fue así que João Gomes, el jefe de redacción de la República, conoció una de las contraseñas de la revolución. "Fue a través del [periodista] Álvaro Guerra que me enteré del 25 de abril. Estaba delante de él [en la sala de redacción] y dijo: "la noche siguiente escucharás la Grândola Vila Morena en la radio..." (João Gomes en entrevista con el autor, Lisboa, 2017)

La euforia que se vivía en las calles se reflejaba también en las portadas de los periódicos, que daban cuenta de los acontecimientos en sucesivas ediciones que iban publicando informaciones actualizadas. Pocos días después, no obstante, se anunciaba un periodo agitado. Maria Antónia Palla, redactora de O Século llustrado, se hace eco de eso mismo, al recordar el reportaje que redactó el día 27:

el primer texto que escribí en libertad acabó por ser cercenado, irreconocible. Ahí me di cuenta de que, si nos habíamos liberado de la censura del régimen, ahora tendríamos que afrontar la censura impuesta por la patronal, por los jefes celosos, por las corrientes ideológicas y por los grupos dominantes (Palla, 2004: 22).

Nuevos y difíciles tiempos se avecinaban. Tras 48 años de censura, los periodistas tendrían oportunidad de aprender a escribir en libertad, pero el periodo revolucionario que sucedió al golpe de Estado va a dejar bien patente el clima de tensión permanente 
que invade las redacciones. Múltiples transformaciones tendrán lugar, acompañando el ritmo de los acontecimientos, reflejando y siendo el reflejo de ellos.

La prensa era vista como un medio privilegiado para hacer llegar determinadas informaciones a la población, lo que explica el combate entablado a la hora de definir la orientación ideológica de los periódicos. Esa y otras tendencias van a ganar protagonismo a lo largo de estos meses.

\section{Del 25 de abril al 11 de marzo: la prensa de Lisboa y la euforia revolucionaria}

Los meses que siguieron al 25 de Abril de 1974 estuvieron marcados por el pulso entre el Presidente de la República, António de Spínola, y la comisión coordinadora del Movimiento de las Fuerzas Armadas 2. En primer lugar, porque Spínola rehusó los nombres propuestos por la comisión coordinadora para el cargo de primer ministro del I Gobierno Provisional, en vías de formación, escogiendo a Adelino da Palma Carlos. Después, porque, mientras la coordinadora del MFA defendía el estricto cumplimiento de su programa en relación a la descolonización, Spínola pretendía una solución federalista, una descolonización de tipo referendario. A grandes rasgos, puede decirse que éste propugnaba una transición gradual, mientras la comisión coordinadora exigía una rápida ruptura con el pasado colonial (Reis, 1994; Ferreira, 2001).

La sociedad portuguesa vivía un momento de gran agitación. Las huelgas y las manifestaciones callejeras se multiplicaban por todo el país, reivindicando mejores condiciones de vida. En ese marco, algunos partidos políticos salen, finalmente, de la clandestinidad y otros van a ser creados, como es el caso del Partido Popular Democrático (PPD, 6 de mayo) y del Centro Democrático Social (CDS, 19 de julio). Si es verdad que en esta primera fase António de Spínola disfruta de una coyuntura favorable, tras la dimisión del primer ministro -después de ver rechazada por el Consejo de Estado una propuesta de aumento de sus poderes y de los del Presidente de la República- la correlación de fuerzas se invierte. Con la toma de posesión del II Gobierno Provisional, liderado por el general Vasco Gonçalves, "el MFA inició su organización autónoma y bajo su iniciativa Spínola hubo de firmar la Ley $7 / 74$, que proclamó el derecho a la independencia de las colonias", asistiéndose, a partir de entonces, a un avance en las negociaciones (Pinto, 2015: 42).

2 Comisión directiva del Movimiento de las Fuerzas Armadas. 
Los enfrentamientos entre el MFA y Spínola se intensifican y éste último pierde poder progresivamente, pasando a estar en una posición bastante débil. "Crecientemente aislado en el aparato de estado y militar, amenazando constantemente con dimitir", Spínola decide "apelar a la mayoría silenciosa, en una tentativa de captar apoyos de los sectores de la derecha civil para revertir la situación" (Rezola, 2007: 105). A pesar de que algunas fuerzas de derecha, como el Partido Liberal y el Partido del Progreso, participan en la preparación de una manifestación de apoyo a Spínola que debería realizarse el día 28 de septiembre de 1974, el plan acaba por fracasar. El objetivo de proclamar el Estado de Sitio no se materializa y el Presidente de la República, presionado por las fuerzas de izquierda y por el MFA, se ve obligado a desconvocar la manifestación, dimitiendo dos días después. Para ocupar su puesto, se escogerá a otro militar, Francisco da Costa Gomes.

Se pueden destacar otras cuestiones en el periodo que se prolonga hasta el 11 de Marzo de 1975: los graves problemas económicos que afrontaba el país, el duro debate en torno a la aprobación de la ley que impuso la existencia de una única central sindical, las diferentes visiones sobre el posicionamiento externo de Portugal, además de las innumerables transformaciones sociales en curso. Se trata, pues, de un periodo bastante agitado -en la calle, en las fábricas y empresas, en los cuarteles, en los órganos gubernamentales y en la política en general-, durante el cual los portugueses verán sucederse dos Presidentes de la República y tres gobiernos provisionales. Como sostiene António Reis, una "nueva contradicción" surgía, en virtud de visiones diferentes acerca del futuro del país: "el modelo democrático-socializante de base parlamentaria, aunque transitoriamente bajo tutela militar, y el modelo revolucionario-socialista, de base populista y bajo la égida de una vanguarda militar". Para este historiador, el Partido Comunista Portugués (PCP) "pasa ahora a exhibir un comportamiento progresivamente más ofensivo y, sobre todo, a explorar las posibilidades de una alianza con todo un sector del MFA, agrupado en torno a Vasco Gonçalves" (Reis, 1994: 24-25).

Éste es el contexto que hace que el periodismo, sus prácticas, rutinas y contenidos se alteren radicalmente. La primera medida que posibilitó esta transformación fue, naturalmente, la abolición de la censura, con la que los periodistas habían lidiado -o intentado sortear- durante casi cinco décadas. La libertad de expresión y de pensamiento estaba, de hecho, prevista en el Programa del MFA (aunque también estuviese previsto un nuevo mecanismo de control, apoyado en una comisión ad hoc para los medios de comunicación, a espera de nombramiento). Pero muchos otros factores, más o menos imprevistos, contribuyen a las transformaciones que van a producirse en la comunicación social.

Junto a los conflictos que se multiplicaron en los medios de comunicación, como las huelgas en los diarios O Século y Jornal do Comércio o los saneamientos políticos que tienen lugar inmediatamente después de la caída de la dictadura, en los que periodistas 
y otros profesionales de la información intervinieron activamente, debía realizarse una adaptación a una nueva realidad político-social $y$, con ella, a una nueva forma de practicar el periodismo. En ese contexto, marcado por enfrentamientos entre los varios sectores que integran las empresas periodísticas, en un ambiente de reivindicaciones y protestas, y en el seno de una sociedad en rápida mutación, los periodistas y los periódicos van a tener que informar de los acontecimientos $y$, a menudo, lidiar con las presiones de los diversos poderes y fuerzas político-militares por hacerse con parcelas de protagonismo en el espacio público.

Por eso, alrededor de los diferentes proyectos políticos para el país -o, si se quiere, de visiones políticas para la prensa del nuevo régimen-se vertebrarán las corrientes en liza entre 1974 y 1975. Compartimos la tesis de Mário Mesquita, para quien existen esencialmente tres corrientes: "los herederos del antiguo régimen, que procuraban retardar el pleno desmantelamiento de los mecanismos censorios", "los defensores de tesis revolucionarias y vanguardistas (...) favorables, en el plano legislativo (por lo menos en el caso del PCP y de sus aliados) a la adopción de formas de censura a posteriori" y "Ios partidarios de una concepción pluralista del sistema de comunicación social" (Mesquita, 1994b: 360-361).

Inmediatamente después del 25 de Abril, el tipo de periodismo que se hacía en algunos periódicos aún refleja mucho de lo que se hacía durante la dictadura. En la redacción del Diário de Notícias, el director adjunto José Carlos de Vasconcelos (desde el 25 de junio) se depara con lo que califica como un "completo desastre", pues, "salvo raras excepciones", se practicaba "un periodismo oficioso, del «ayer se realizó», sin pulso, sin fuerza, muy burocrático y muy vinculado obviamente al régimen" (José Carlos de Vasconcelos en entrevista con el autor, Lisboa, 2012). Lo cierto es que se trataba de "una experiencia nueva. Nosotros siempre habíamos escrito hasta entonces pensando que aquel texto tenía que pasar por la censura", como recuerda Maria Antónia Palla, entonces en O Século Ilustrado (Maria Antónia Palla en entrevista con el autor, Lisboa, 2013). "Los periodistas de mi generación estaban especialmente ansiosos por la libertad", recuerda Maria Teresa Horta, en ese momento en la A Capital (Maria Teresa Horta en entrevista con el autor, Lisboa, 2011). En los meses siguientes, se van incorporando nuevos redactores, al tiempo que se adoptan nuevas formas de organización de las redacciones, de trabajo y de producción periodística.

Tras años de contención en la escritura, las redacciones conocen una "explosión" de ideologías y la posibilidad de implicarse activamente en la política (con reflejos casi inmediatos) fue aprovechada por muchos periodistas y otros profesionales de la comunicación. Sólo más tarde, pasados los meses de revolución, le sería posible a la profesión "extenderse a nuevas capas sociales, buscar nuevos públicos, reconstituirse como conjunto profesional, establecer su compromiso moral, intentar afirmar su 
jurisdicción, acompañando y estimulando la formación de un universo de la información" (Garcia, 2009: 35).

Durante el periodo revolucionario las redacciones van admitiendo periodistas, pero "los criterios partidarios se sobrepusieron a cualquier referencia de otro tipo". Las cualidades profesionales quedaron en segundo plano con respecto a la "militancia política", produciéndose asimismo la entrada de periodistas procedentes de las antiguas colonias africanas, "que serían, en su conjunto, un contrapeso a la izquierdización resultante de los reclutamientos" (Mesquita, 1994b: 364). Naturalmente, habrá habido excepciones a esta tendencia. "La política y el debate ideológico estaban en el centro de la vida y eran el alma de la información periodística, lejos, aún, de las prácticas entronizadas de los principios de objetividad y de contradicción", observa João Figueira (2012: 8). Era el periodismo absorbiendo y siendo absorbido por su contexto, acompañando los acontecimientos, implicándose en ellos y dejando la reflexión sobre las cuestiones éticas, deontológicas y otras reglas de la profesión para más tarde. En este contexto, era "imposible" mantener la neutralidad periodística y la situación en que los propios periodistas se encontraban explica, en gran medida, esa realidad:

la habituación a la censura, que forzaba a un lenguaje cifrado, la inexistencia de estudios reglados en comunicación o en periodismo, la ausencia de homogeneidad (y esto no sólo a nivel político propiamente dicho, sino en el sentido de un mínimo de entendimiento común sobre qué es y cómo se practica el periodismo) en los equipos de redacción heredados del periodo anterior al 25 de Abril (Mesquita, 1994a: 363-364).

Ante estos factores, los relatos de los propios periodistas subrayan algo que surge como evidente: la situación era tal que se estaba lejos de poder poner en práctica el ideal de periodismo que, con mayor o menor diferencia, todos ellos defienden. Había una "casi unanimidad de los directores y periodistas acerca de su papel de protagonistas políticos", mantiene Kenneth Maxwell (1983: 15).

Daniel Ricardo, editor de la A Capital y, más tarde, del Diário de Notícias, recuerda que, "después del 25 de abril, los periodistas se dividieron. Algunos fueron a algunos partidos, otros fueron a otros... comenzaron grandes luchas políticas" (Daniel Ricardo en entrevista con el autor, Lisboa, 2011). En ese momento en la República, Diogo Pires Aurélio recuerda que en "periodismo en ese momento" la discusión era 100\% política", ya que "no había obstáculos para la independencia más profesional". El periodismo "se asumió revolucionario" (Diogo Pires Aurélio en entrevista con el autor, Lisboa, 2017).

Otro periodista, Luís de Barros, subjefe de redacción del Expresso y, después, director del $D N$, refuerza la idea de que "se hacía política en todas partes" y, por eso, "los periódicos, evidentemente, eran, por excelencia, medios para la comunicación política" (Figueira, 2007: 198). Con algunas variaciones, la verdad es que esta tendencia hacia la 
exposición de las opciones políticas en el periodismo fue una constante, formando parte de las discusiones diarias en las redacciones.

El ritmo de la revolución se aceleraba de manera vertiginosa. El material periodístico abundaba, lo que suponía también un enorme reto para los periodistas, además de acarrear obvias responsabilidades. "Por primera vez las redacciones se vieron enfrentadas a la necesidad de cubrir una cadencia informativa constante, por oposición al marasmo vivido anteriormente". De resultas de lo cual, "los periódicos reflejaron en sus páginas el clima de sobresalto que se apoderó del país", sostiene Helena Lima (2008: 144).

Lidiar con el "poder" -con los diversos poderes- constituía, también, un gran desafío. No sorprende, por lo tanto, el hecho de a menudo se diera pábulo a rumores e informaciones no confirmadas, inclusive en titulares de prensa. Maria João Avillez, redactora del Expresso, "pasaba los días en los cuarteles o en el Consejo de la Revolución, que eran los sitios donde generalmente el dr. Balsemão [director] me mandaba. Era preciso estar en todas partes al mismo tempo y nosotros lo estábamos" (Avillez, 2016: 269). José Jorge Letria (República) va más lejos, recordando que había "información en exceso y al mismo tiempo", siendo "contradictoria y poco organizada" (Letria, 2013: 193). En el mismo sentido, José Carlos de Vasconcelos (Diário de Notícias) recuerda que "era el tiempo de los hombres sin sueño. Cerrábamos el periódico hacia las tres, cuatro de la mañana... Una noticia que era cierta a la una, a las dos ya no lo era" (José Carlos de Vasconcelos en entrevista con el autor, Lisboa, 2012).

Otro periodista, Dinis de Abreu, entonces en el Diário Popular, hace una vívida descripción, que coincide con los recuerdos de sus colegas, afirmando que, en el fondo, las redacciones no eran más que un espejo de lo que se vivía en el país:

El ambiente revolucionario había transbordado a las redacciones, que respiraban el día a día, entre noticias contradictorias de golpes y de contragolpes, de cuarteles en armas, de aviones sobrevolando Lisboa, de comandos sublevados, de fuerzas populares en las calles, sin rey ni Roque. Pasé muchas horas agarrado a teléfonos haciendo contactos, aclarando rumores que desaguaban, a cada momento, en la redacción (Abreu, 2016: 276).

Uno de los "Capitanes de Abril", Vasco Lourenço, se refiere a esa realidad, confirmando los vínculos que existían entre periodistas y fuentes de poder, en el sentido de que éstas buscaban obtener una posición destacada en el espacio mediático: “Por aquel entonces toda la gente conspiraba y contra conspiraba. Toda la gente tenía sus conexiones en los periódicos para hacerles pasar las cosas que les interesaba [comunicar] y los periódicos estaban interesados" (Vasco Lourenço en entrevista con el autor, Lisboa, 2012).

El resultado del ritmo frenético y "apasionado" de trabajo de los periodistas estaba lejos de ser lo más útil para los lectores en aquel momento, que es, en el fondo, la misión de 
un periódico: informar. A menudo no estaba clara la distinción entre artículos de opinión e informativos. Otra característica del periodismo de este periodo era que la aplastante mayoría de los artículos no estaban firmados (excepto los artículos de opinión, aunque no siempre). Se añade la dificultad en interpretar textos poco claros, bastante adjetivados y en que los leads no respondían a las cuestiones fundamentales. De acuerdo con Francisco Rui Cádima, en los primeros meses que siguieron al golpe militar, el tono de los órganos de comunicación no era muy diversificado:

Los media, de alguna manera, reflejaban un cierto servilismo político al MFA, y con una alta dosis de paternalismo, en una primera fase, apelaban constantemente a la necesidad de que el MFA protegiera a la comunicación social de los discursos de la «reacción», especialmente en los meses que anteceden al 28 de Septiembre de 1974 y muy en particular en el contexto de la manifestación de la mayoría silenciosa (Cádima, 2001: 328).

Por otro lado, las ventas de los periódicos subían exponencialmente y en los quioscos aparecían nuevos títulos, de los géneros más diversos, por más que la duración de muchos de ellos con frecuencia fuese reducida. "El bullicio periodístico se extendió a las calles de Bairro Alto y Chiado, donde estaban las salas más grandes de la redacción de Lisboa y donde "se respiraban los periódicos y los periodistas". Todos los días yo salía de la A Capital, Chiado abajo, para la [cafetería] "Brasileira”. Había gritos de los repartidores de periódicos por todos los lugares...", dice Fernando Gaspar (Fernando Gaspar en entrevista con el autor, Lisboa, 2017).

Importa destacar que la politización de los contenidos de la prensa es también, en no pocas ocasiones, causa o consecuencia (según los casos) de los conflictos que van surgiendo en el interior de los propios periódicos, entre administradores, directores, periodistas y otros trabajadores. Las divergencias sobre todo tenían que ver con el "reparto de poderes en el interior de las empresas periodísticas". Además de los periodistas, otros trabajadores reclaman poder decisorio acerca de los contenidos que debían publicarse. Por su combatividad destaca, en este dominio, el sector gráfico. "Las células comunistas y de la extrema izquierda revolucionaria de las tipografías consiguieron, en ciertos casos, imponer su voluntad en las empresas", sostiene Mário Mesquita (1988: 93 y 104).

La Ley de Prensa, otra medida prevista en el Programa del MFA, empezaba a ser preparada, por lo que sus perfiles aún estaban por delimitar. Se trataba, esencialmente, de decidir a quién pertenecía la función de definir el contenido de un periódico. En el Diário de Lisboa, por ejemplo, "la dirección de Ruella Ramos fue aceptada con «agrado», pero los trabajadores asumieron el control de la producción del periódico y, merced a la unión redactores-técnicos, el PCP y la extrema izquierda aumentaban su influencia" (Matos, 2016: 139). En diciembre de 1974, en el semanario Expresso, se plantea la hipótesis de un boicot de quiosqueros a la venta del semanario, aparentemente debido 
"a los márgenes de lucro" de los vendedores. La atribución de "implicaciones políticas" al boicot es inmediata, obligando a una intervención del gobierno (Relatório do Conselho de Imprensa, 1979: 32). En O Século, los plenarios eran frecuentes y el clima iba subiendo de tono, hasta el punto de que en varias ocasiones se produjeron enfrentamientos entre trabajadores, debido a visiones distintas sobre cuál debía ser su línea editorial.

La importancia de las Comisiones de Trabajadores (CT) era evidente. Eso no era únicamente característico de las empresas de comunicación, extendiéndose a los más diversos campos. Un poco por todas las empresas del país, las CT asumen un papel determinante, esforzándose para alcanzar los objetivos del colectivo, muchas veces en plenarios de trabajadores, con votación a mano alzada. Las reivindicaciones versaban sobre condiciones y derechos laborales (se produjeron mejoras significativas en el sector de la prensa, con la aprobación de un nuevo Contrato Colectivo de Trabajo, por ejemplo) pero también a prácticamente todos los temas que afectaban a las empresas. João Candeias, tipógrafo y miembro de CT del Diário de Notícias, revela los logros del trabajo realizado, el resultado del poder de reclamo que los trabajadores pasaron a tener:

Fui tan lejos que llegué a tener más poder que el presidente de la Junta de Directores, porque lo que nosotros decidíamos era prácticamente irrefutable. Nos reunimos por los repartidores de periódicos. Los nuestros días libres eran hechos rotativamente y empezamos a tener días libres fijos para que no fuéramos lastimados (João Candeias en entrevista con el autor, Lisboa, 2012).

Estas Comisiones, así como los Consejos de Redacción que entre tanto fueron creados, pasan a "ejercer una influencia determinante sobre lo que se publica" (Relatório do Conselho de Imprensa, 1979: 15), mientras los administradores y los propios directores de los periódicos pierden poder decisorio. Muchos conflictos tuvieron inicio o se agudizaron por este motivo. En realidad, era inexistente la regulación sobre esta cuestión crucial en la rutina de un periódico, lo que vendría a corregirse en febrero de 1975, con la aprobación de la Ley de Prensa. 


\section{Tras el 11 de marzo: los periódicos y el país en ebullición}

El 12 de marzo de 1975, el diario República informa en primera plana: "La institucionalización inmediata del MFA y la creación del Consejo de Revolución (CR) 3 decisiones tomadas en la Asamblea de Militares" 4 . Dos días después, el titular del Diário de Lisboa anuncia la nacionalización de la banca5. Estas son, sin duda, dos cuestiones fundamentales en el nuevo contexto revolucionario.

No cabe aquí describir los acontecimientos que rodean la tentativa de golpe del $11 \mathrm{de}$ Marzo6, sino apenas hacer referencia a su obvio significado en términos de alteraciones político-militares en el proceso revolucionario. Como ya se señaló, el poder militar se institucionaliza, a través de la creación del Consejo de la Revolución, pasando ahora a ser presentado como el "motor" de la revolución. Las primeras y más emblemáticas medidas tomadas por el CR son "en el campo-económico la nacionalización de la banca y de los seguros" $y$, en el plano militar, las "destinadas a expulsar a los implicados en el golpe y a reorganizar la estructura militar, para que la jerarquía formal coincidiese con la revolucionaria" (Cervelló, 1993: 229).

Medidas que representaban, sin duda, un viraje a la izquierda (otras nacionalizaciones van a tener lugar), que no era, en absoluto, ajeno a la preponderancia del sector próximo al primer ministro Vasco Gonçalves (por lo que pasó a ser conocido como «gonçalvista») en las estructuras político-militares y al creciente protagonismo del Partido Comunista en la escena política. Se producían por entonces las primeras ocupaciones de tierras en el Alentejo, ganando terreno el llamado "poder popular", que se traducía "en el control por autogestión de centenares de pequeñas fábricas y en la ocupación de casas abandonadas, llevada a cabo por grupos de particulares con el apoyo del COPCON7 y de partidos de extrema izquierda" (Reis, 1994: 30). En abril, se firma el Pacto entre el MFA y los principales partidos políticos, realizándose, a fines de ese mismo mes, las primeras elecciones libres y democráticas - para la Asamblea Constituyente, que tenía como misión elaborar la nueva Constitución portuguesa- con una victoria del PS (Partido

\footnotetext{
3 Creado el 14 de marzo de 1975, el Consejo de la Revolución, constituido por militares, ejerció las competencias de la JSN, del Consejo de Estado y del Consejo de Jefes de Estado Mayor. En la práctica, significaba la institucionalización del MFA.

4 República, 12 de marzo de 1975.

5 Diário de Lisboa, 14 de marzo de 1975.

6 Se trató de una intentona fallada de golpe de Estado, desencadenada por el ex Presidente de la República, general António de Spínola.

7 EI COPCON (Comando Operacional del Continente) fue un órgano militar que tenía, entre sus funciones, intervenir en el mantenimiento y restablecimiento del orden público y apoyar a las autoridades civiles.
} 
Socialista), con un $37,9 \%$ de los votos, mientras que el PCP $(12,5 \%)$ quedaba por detrás del PPD (26, 9\%).

Legitimados por el resultado obtenido en las elecciones, los socialistas van a potenciar su popularidad en las calles mediante la organización de grandes manifestaciones y mítines. Los incidentes en el Estadio 1으 de Maio, donde a algunos dirigentes del partido se les impide llegar a la tribuna y discursar, acaban por dotar de una "nueva dimensión y dramatismo" al "pulso entre la vía revolucionaria y la vía electoral, protagonizado en términos políticos por el PCP y sus aliados y por el PS" (Ferreira, 2001: 170).

Se añaden los problemas que rodearon el cierre del periódico República, que llevarán tanto al líder del Partido Socialista, Mário Soares, como a los restantes ministros socialistas a abandonar el gobierno. Más tarde, el PPD tomará la misma actitud, haciendo caer el IV Gobierno Provisional. La agitación en las calles sube de tono, asistiéndose a una oleada de asaltos a sedes de partidos de izquierda y de sindicatos, en un clima de antesala de guerra civil. En pleno "Verano Caliente», el campo militar se encuentra profundamente dividido y los varios documentos producidos en ese contexto, que apuntan a rumbos distintos para el país, son, a ese respecto, ejemplificativos (Rezola, 2007: 221-227).

Contra la posición del ala militar moderada o de partidos como el PS y el PPD, el Presidente de la República acabará por llamar a Vasco Gonçalves para liderar el muy contestado (y corto) V Gobierno Provisional. Es en este contexto de profundas escisiones en el seno del ejército, acompañadas de una gran agitación social y enfrentamientos políticos, con el PCP pasando de una posición de preponderancia a verse contestado por un PS cada vez más fuerte, cuando el sector de la Información atraviesa su periodo más tenso y agitado.

Las ya mencionadas nacionalizaciones del post 11 de Marzo tienen efectos inmediatos en la prensa nacional. Gran parte de las empresas que pertenecían al sector bancario acaba por ser, por esa vía, expropiadas y los periódicos no escapan a la regla. De depender de grandes grupos económicos pasan a pertenecer al sector público el Diário Popular y el Jornal do Comércio (Grupo Quina, del Banco Borges \& Irmão); A Capital (también Grupo Quina y, después, Banco Espírito Santo \& Comercial de Lisboa); $O$ Século, incluyendo Vida Mundial, el Século Ilustrado y Modas \& Bordados (grupo económico de Jorge de Brito, del Banco Internacional Português) y el Diário de Lisboa, una vez que un tercio de su capital pertenecía al Banco Nacional Ultramarino y sus elevadas deudas a la banca hicieron que pasase a ser dominado por el Estado. En el caso 
del Diário de Notícias, éste ya se encontraba, por vía indirecta, integrado en el sector público8.

De la prensa lisboeta, los únicos títulos que siguen siendo privados son el diario República y el semanario Expresso. Consecuencia indirecta de lo que había sucedido en el sector bancario, era obvio que una prensa bajo el control del Estado implicaba, desde luego, que el gobierno y el Consejo de la Revolución se vieran tentados a imponer determinados nombres para los cargos de administración y dirección de las diferentes cabeceras. ¿Habrían "aprovechado" aquéllos su posición privilegiada para colocar a personalidades de su cuerda política en los periódicos? ¿Tendrían estas figuras así colocadas motivaciones políticas en el ejercicio de sus nuevas funciones? Y, en los casos en que no hubo alteraciones en esos órganos, ¿se produjeron cambios en los contenidos? La bibliografía existente sobre el asunto parece llevarnos a responder afirmativamente a esas cuestiones.

De acuerdo con el informe del Consejo de Prensa, en este periodo se acentúa "la influencia y la manipulación partidaria en la prensa, en especial por parte de elementos próximos al Partido Comunista y a organizaciones de extrema izquierda" (Relatório do Conselho de Imprensa, 1979: 43). Varios autores admiten que se trataba, efectivamente, de una estrategia previamente definida y estructurada por esas fuerzas políticas. Francisco Rui Cádima, por ejemplo, defiende que "las escaladas comunista e izquierdista en el asalto al poder - unas veces coincidentes, otras no- estaban lanzadas y estratégicamente conquistaban las primeras líneas de batalla", que eran los media (2001: 331).

De acuerdo con Carlos Gaspar, fue precisamente el "peso en los medios de comunicación de masas", conjuntamente con otros factores, lo que hizo que el PCP se impusiera "como el más fuerte de los partidos políticos" en este periodo (Gaspar, 2010: 550). Para Medeiros Ferreira, el Partido Comunista condujo su acción con miras a alcanzar objetivos concretos, en particular "controlando una vasta gama de órganos de comunicación social, donde llegan a practicar actos de censura conocidos" (Ferreira, 2001: 206).

No obstante, como también subraya Mesquita, los efectos del control de los medios de comunicación estuvieron lejos de lograr el éxito deseado. Fueron, como se sabe, limitados. Uno de los ejemplos que este autor presenta es el del resultado de las ya mencionadas primeras elecciones libres (para la Asamblea Constituyente): "a pesar de su aplastante preponderancia en la información, el PCP y sus aliados fueron nítidamente derrotados en las elecciones en beneficio de las corrientes defensoras de la democracia

8 En la práctica, el periódico pertenecía al Estado, ya que la Caixa Geral de Depósitos era titular de la empresa "Portugal e Colónias" y ésta de la Empresa Nacional de Publicidade, propietaria del Diário de Notícias. 
representativa" (Mesquita, 1994b: 365). La coyuntura creada por el nuevo momento político-militar potenció prácticas y posicionamientos ya verificados en los últimos meses, agravándolos. El clima extremado y polarizado había llegado, inevitablemente, al sector de la información, alcanzando su punto más crítico en el verano. Veamos lo que sucede en algunos diarios.

El Diário de Notícias vuelve a ser un ejemplo paradigmático. En un plenario de trabajadores, se retira la confianza al director del periódico, bajo la acusación de que, en su editorial, éste había condenado las propuestas, supuestamente realizadas en la Asamblea del MFA, de fusilamiento de los militares implicados en el 11 de Março9. José Ribeiro dos Santos y su adjunto, José Carlos de Vasconcelos, acaban por dimitir, siendo sustituida también la administración. El gobierno nombra, ese mismo mes de marzo, una nueva administración y dirección. Ésta última pasaría a estar formada por Luís de Barros y José Saramago, dos nombres próximos al PCP, y el segundo de ellos militante de ese partido.

Además nuevos periodistas van a llegar a la redacción, como es el caso de José Jorge Letria (procedente del República), que recuerda la acción del director-adjunto y el posicionamiento ideológico que imprimió al periódico: "Saramago, como ya antes había sucedido en el Diário de Lisboa, estaba muy poco presente en la vida de la redacción, pero era muy activo en la definición de su línea política, teniendo a su cargo la redacción de los editoriales que reflejaban fielmente las posiciones del PCP” (Letria, 2013: 230). Su colega, Mário Contumélias, va más lejos, llegando a considerar que "el Diário de Notícias era, a partir de un determinado momento, el periódico del Partido Comunista" (Mário Contumélias en entrevista con el autor, Lisboa, 2011). Pero, como observa João Figueira, los contenidos del periódico no se limitaban a destacar el papel del Partido Comunista en la revolución, pues el diario "orientó toda su estrategia noticiosa y de opinión, adoptando como suya toda la acción política de Vasco Gonçalves [primer ministro] y de las fuerzas identificadas con él y con el PCP" (Figueira, 2007: 125).

En cuanto al contenido, los investigadores británicos Jean Seaton y Ben Pimlott nos dan una percepción clara del efectivo posicionamiento político-ideológico del periódico, comparándolo con la postura de servilismo que había asumido en la dictadura:

La situación más dramática (pero también la más previsible) fue la del Diário de Notícias, el matutino de Lisboa de mayor circulación, que siempre había seguido las posiciones del antiguo régimen. En 1974, el Diário de Notícias transcribía fielmente los procedimientos del congreso de la Unión Nacional Portuguesa (el partido del gobierno). En abril de 1975, informaba con la misma calma y tono referencial sobre los encuentros de la central sindical comunista, Intersindical. Los

9 Cf. Diário de Notícias, 14 de marzo de 1975. 
mismos periodistas escribían de la misma manera, con la misma aceptación de la autoridad política prevaleciente (Seaton y Pimlott 1983, 102).

Pero el $D N$ era también un periódico afectado por un clima tenso, resultado de conflictos entre trabajadores, directores y administradores, como bien ilustra el «caso de los 24», que llevará a la depuración política de un grupo de periodistas, en un proceso muy mediatizado y politizado, que se arrastra más allá del 25 de Noviembre10 (Gomes, 2014). En el vespertino A Capital, los problemas también se acumulan. En primera instancia, por la tensión que rodeó la censura de un artículo de la escritora Natália Correia, que desembocó en la dimisión, por solidaridad, del director David Mourão Ferreira, quien unos meses después, en diciembre, fundará $O$ Dia.

Para el Diário de Lisboa se nombra un nuevo administrador (militar), en representación del Estado. De acuerdo con el director de entonces, se asistió a "perturbaciones" y a "luchas partidarias por la conquista del poder dentro del periódico" y "todo eso amargó la alegría de la libertad y debilitó aún más a un diario que ya había entrado frágil en el régimen democrático" (Ramos, 1994: 287). El vespertino adoptará una "imagen demasiado radical, que alejó a una parte considerable de sus lectores tradicionales" y el Diário Popular, que ve también nombrado un nuevo administrador (militar), "se hiperpolitizó" y "se apartó de la vocación "popular» implícita en el título" (Mesquita, 1994b: 387), después de ver cómo su administración era sustituida por exigencia de los trabajadores, que llegan a movilizarse em piquetes de vigilancia11. Por su parte, O Século vive momentos dramáticos, multiplicándose los plenarios. La dirección del periódico es sustituida, algunos trabajadores son suspendidos, se realiza una manifestación de trabajadores y se publica un periódico de lucha, siendo elegido un tipógrafo, Francisco Lopes Cardoso, como director (Gomes, 2018: 52-54). Fuera del dominio del Estado estaba el Expresso, que "atacó y criticó sin recelo al primer ministro Vasco Gonçalves", siendo "la más influente y escuchada voz del centro-derecha" (Figueira, 2007: 132).

La verdad es que el periodismo se había enredado, cada vez más, en la revolución. La "vocación" de los diarios no era "explicar o persuadir, sino agitar y movilizar" y la "información era servida "en bruto", mal digerida, tal como salía de los palacios gubernamentales, de las sedes partidarias o de los cuarteles más influenciados por las doctrinas políticas" (Mesquita, 1994: 363-364). En agosto de 1975, un análisis del Presidente de la República presenta un panorama desolador de la comunicación social portuguesa: "Puede comprobarse que, en términos generales, la información hablada y

10 A pesar de las dudas que todavía existen sobre el desarrollo de este episodio, los acontecimientos del 25 de Noviembre de 1975 significan el punto final del proceso revolucionario, marcando el inicio de un nuevo momento político: el de la institucionalización de la democracia en Portugal.

11 O Primeiro de Janeiro, 9 de abril de 1975. 
escrita ha contribuido, en gran medida, al agravamiento de las tensiones políticas hoy vividas en el país, poniendo en riesgo la supervivencia de la propia revolución" 12.

Se mantiene el ritmo de trabajo. "El equipo de redacción continuaba por la noche, porque había reuniones de AMF que no terminaban temprano. Periodistas que fueron al [bar] "Procópio"... Los líderes políticos y militares que, a las 3 ó 4 de la mañana, fueron a hablar al periódico. A menudo recuerdo haber recibido miembros del Consejo Revolucionario o altos rangos militares a las 4/5 de la mañana", dice Diogo Pires Aurélio, quien dejó la República para fundar el periódico Jornal Novo (Diogo Pires Aurélio en entrevista con el autor, Lisboa, 2017). La movilización de los trabajadores, especialmente del sector gráfico, a través de las respectivas Comisiones y de la convocatoria de plenarios, se mantiene; en ciertos casos, de hecho, su poder dentro de los periódicos aumenta considerablemente. En ocasiones, los lectores se manifiestan contra los contenidos producidos, ya sea por medio de cartas enviadas a las redacciones, como de protestas públicas como quemas de fajos de periódicos o impedimento de su distribución.

Con respecto a la prensa en la fase posterior al 11 de Marzo, no podríamos dejar de mencionar el "Caso República", paradigmático porque afecta a un periódico con una conocida tradición de oposición al Estado Novo. Desde principios de mayo el clima en el República era tenso. Primero, porque el sector gráfico se opuso a la contratación de dos nuevos periodistas, acusando a la dirección de haber realizado una contratación partidaria. Después, una nueva protesta se produce cuando la administración pide la dimisión del director comercial, Álvaro Belo Marques. El 19 de mayo, la Comisión de Trabajadores decide suspender a la dirección (Raul Rêgo y Vítor Direito) y al jefe de redacción (João Gomes), acusándoles de estar al servicio del PS (no era un secreto que Rêgo y Gomes eran fundadores y militantes del Partido Socialista), procediendo a la ocupación de las instalaciones del periódico.

En el editorial que publican ese día, los trabajadores se refieren a ellos como "figuras marcadamente antifascistas, pero también marcadamente partidarias" 13 . Plantean a Rêgo y a los otros afectados que abandonen el periódico, lo que estos rechazan tajantemente. A su lado tenían a la mayoría de la redacción (22 de los 24 periodistas) que se opone a la decisión de la Comisión de Trabajadores. En un comunicado, dicen estar ante una maniobra destinada a "callar una -y probablemente la más fuerte- de las voces libres de este país" 14 . Encerrado en su gabinete, Raul Rêgo telefonea a Mário Soares, quien se desplaza a la sede del periódico en la Rua da Misericórdia junto con otros nombres relevantes del Partido Socialista y numerosos ciudadanos para

\footnotetext{
12 O Primeiro de Janeiro, 28 de agosto de 1975.

13 República, 19 de mayo de 1975.

14 Jornal Novo, 20 de mayo de 1975.
} 
manifestarse contra la ocupación del República. Se canta el himno nacional y se gritan consignas como "Este periódico no es de Cunhal [líder del PCP]". El caso se trasladaba al plano de los enfrentamientos entre el PS y el PCP, situación que aprovecha Soares para responsabilizar a los comunistas de los problemas que se estaban produciendo 15 .

Los acontecimientos alrededor del caso se suceden: la edición del día 19 sale a despecho de la dirección y el nombre de Álvaro Belo Morais aparece en lugar del director; el Ministro de Comunicación Social, Correia Jesuíno, intenta, sin éxito, resolver el conflicto; el COPCON precinta las instalaciones del República; la mayoría de la prensa internacional interpreta el caso como un ataque del PCP a la libertad de expresión (una excepción en este sentido será Le Monde); el PS suspende su participación en reuniones del Consejo de Ministros hasta que se resuelva el problema y, el 10 de julio, el periódico reaparece con un nuevo director (coronel Pereira de Carvalho), nombrado por el Consejo de la Revolución, a propuesta de los trabajadores. Es decir, a pesar de que el gobierno había ordenado la devolución a la administración y dirección, tal como se establecía en la Ley de Prensa recién aprobada, el COPCON no cumple la orden y entrega el República a la Comisión de Trabajadores. Como apunta Joel da Silveira, salía victoriosa la "concepción de la prensa popular en antítesis con todo lo que no fuese revolucionario, incluyendo las corrientes del socialismo democrático, cimentada por la unidad antifascista y al servicio de los trabajadores, en ese caso encarnados en los trabajadores gráficos del República" (Silveira, 2011: 135).

Este es, indiscutiblemente, uno de los casos más mediáticos y relevantes del proceso revolucionario. Cuestiona la Ley de Prensa, que daba la razón a la dirección y administración del jornal, pero, sobre todo, crea -o contribuye fuertemente a crearuna crisis en el gobierno. Tras la salida a los quioscos del República, bajo la dirección de Pereira de Carvalho y con una nueva redacción, los socialistas emiten un comunicado donde recuerdan que "el Consejo de la Revolución, así como el Consejo de Prensa, habían decidido que el jornal debería ser entregado a la dirección, y que Costa Gomes había afirmado públicamente que el caso ya estaba resuelto" (Castaño, 2013: 320). Anunciaban la salida de sus ministros y secretarios de Estado del IV Gobierno Provisional, que pocos días después es secundada por los del PPD.

Varios aspectos se entrelazan en torno a este conflicto y sus diferentes matices, que implican cuestiones laborales, pero también político-ideológicas, que sitúan al PS, al PCP y a la extrema izquierda en el centro de la polémica. No obstante, algo es innegable:

A través del caso República se discutieron la Ley de Prensa, el control obrero, la organización de la empresa periodística, los poderes del director y del consejo de redacción, el derecho a la información, la unidad de la izquierda portuguesa y

15 Sobre el «Caso República» cfr., entre otros, Mesquita 1994a. 
francesa, el eurocomunismo, los regímenes del Este Europeo" (Mesquita, 1994a: 508).

Ante un panorama de periódicos nacionalizados como el que se ha descrito, los problemas en el diario privado República y un telón de fondo político-social agitadísimo, va a ser el sector de la prensa privada el que llevará al Consejo de Prensa a considerar que el "monolitismo de la prensa no es total" (Relatório do Conselho de Imprensa, 1979: 44). Efectivamente, la creación de nuevos periódicos a partir de abril de 1975 - entre los cuales destacamos el Jornal Novo, o Jornal, el Tempo y A Luta - significó, efectivamente, una alternativa a la prensa estatalizada. João Gomes, el fundador de la $A$ Luta, recuerda que el grupo de periodistas que formaron la sala de redacción "vivía constantemente con miedo de que el Partido Comunista pudiera ganar" (João Gomes en entrevista con el autor, Lisboa, 2017). Fue una lucha en la que se enfrentaron muchas posiciones y estábamos del lado de aquellos que defendían la democracia". José Sasportes, quien fundará el Jornal Novo, destaca lo que estaba en juego en el nuevo proyecto: "Decidimos hacer un periódico con una perspectiva progresiva, pero completamente independiente de cualquier partido" (José Sasportes en entrevista con el autor, Lisboa, 2017).

Estos periódicos, bajo diferentes aspectos, innovadores y osados, sin dejar de ser también polémicos, de implicarse y tomar partido en el curso de los acontecimientos y de asumirse, sin equívocos, como defensores de la legalidad democrática como vía política para el futuro del país (Gomes, 2018). Además de significar una alteración en el panorama de la prensa de la época, estos nuevos diarios contribuyeron también a cambiar la situación política. Como defiende António Telo, "las primeras grandes derrotas del gonçalvismo se producen justamente en la comunicación social, tanto por el tiro en el pie que suponen los asaltos lanzados al República y a la RR [Rádio Renascença], como por el tiro en la cabeza que representan los nuevos periódicos" (2007: 130).

Serían precisamente estos periódicos los que acabarían por ser los vehículos de las informaciones sobre el 25 de Noviembre, ya que se suspendió la publicación de la prensa nacionalizada, que tardará algún tiempo en regresar a los quioscos. Llegaba a su fin la revolución portuguesa y, en lo tocante a los periódicos, eso significaba nuevas alteraciones en las administraciones, direcciones e incluso a nivel de las redacciones.

\section{Conclusión}

En el período revolucionario hubo, entonces, una "explosión" de información política. Todo estaba cambiando en Portugal y, en esa medida, también los periódicos y el periodismo acompañaban la tendencia. 
El periodismo - y, particularmente, el periodismo político - se mantuvo en las antípodas del desiderátum de neutralidad que caracteriza la profesión. Esto, en parte, podrá deberse al hecho de que muchos periodistas eran, simultáneamente, militantes partidarios. Pero también, y quizás sobre todo, porque, independientemente de los proyectos que defendían, todos deseaban participar en la construcción de un nuevo régimen para el país y, en esa medida, participar en la revolución.

Se trataba, como algunos autores han señalado, de un periodismo "revolucionario" y "militante", en el sentido de que pretendía agitar, movilizar y, en muchos casos, hacer política. Las tiradas aumentaban y los periódicos criticaban y apoyaban, sin pudor, las fuerzas en presencia. Los editoriales asumen particular protagonismo como textos de agitación política. Tal como había sucedido durante la dictadura, extensos documentos de texto corrido, con frecuencia publicando literalmente comunicados de órganos de Poder, se presentaban sin ningún tipo de interpretación

A menudo, no estaba clara la distinción entre artículos informativos y de opinión. Muchos de ellos ni siquiera estaban firmados. Era habitual que un texto presentado como una noticia estuviera glosado con comentarios que ponían en evidencia la posición del periodista sobre el tema tratado, siendo también frecuente el recurso a la adjetivación. En cuanto a la cantidad de acontecimientos e informaciones, era avasalladora, lo mismo que el ritmo de trabajo. Todas estas cuestiones se verán agravadas después del 11 de Marzo y de la nacionalización de gran parte de los periódicos. Los intentos de control de los órganos de comunicación se intensifican, ocurriendo episodios que han quedado para la historia de este periodo y que ilustran bien el clima de radicalización y polarización que se vivía (casos República y Diário de Notícias, entre otros) dentro y fuera de las empresas del sector.

En resumen, parece evidente, no sólo en la bibliografía existente como también en los relatos que hemos recogido de varios periodistas, que los periódicos participaron en los juegos de luchas y en el clima de enfrentamiento que caracterizaron el proceso revolucionario portugués. Para más tarde quedaron los debates sobre las fronteras entre el periodismo y la política, la información y la opinión. Para ello, fue esencial la normalización democrática, una Constitución con un articulado importantísimo dedicado a la comunicación social, un código deontológico y una renovación en el panorama mediático nacional, lo que sólo sucederá después de 1976. 


\section{Referencias bibliográficas}

ABREU, D. (2016): "O 25 de Novembro e os media”, en Barreto, A. y Cruz, M. B. et al. (org.), O 25 de Novembro e a democratização portuguesa, Lisboa, Gradiva, pp. 275-281.

AURÉLIO, D. P. (2017): Entrevista dada a Pedro Marques Gomes. Lisboa.

AVILLEZ, M. J. (2016): “O fim da revolução” en Barreto, A. y Cruz, M. B. et al. (org.), O 25 de Novembro e a democratização portuguesa, Lisboa, Gradiva, pp. 269-274.

AZEVEDO, M. (2010): Memória de uma mulher de Letras, Porto, Afrontamento.

BAPTISTA, C. (2019): "A invenção da televisão revolucionária - a RTP durante o PREC (1974-1975)" en Media \& Jornalismo, no 35, Vol. 19, № 2, Lisboa, ICNOVA, pp. 237247.

CÁDIMA, F. R. (2001): “Os "Media» na Revolução (1974-1976)" en BRITO, J. M. (coord.), O País em Revolução, Lisboa, Editorial Notícias, pp. 321-358.

CANDEIAS, J. (2012): Entrevista dada a Pedro Marques Gomes. Lisboa.

CASTAÑO, D. (2013): Mário Soares e a Revolução, Lisboa, Dom Quixote.

CERVELLÓ, J. S. (1993): A Revolução Portuguesa e a sua Influência na Transição Espanhola (1961-1976), Lisboa, Assírio e Alvim.

CONTUMÉLIAS, M. (2011): Entrevista dada a Pedro Marques Gomes. Lisboa.

FERREIRA, J. M. (2001): Portugal em Transe en Mattoso, J. (dir.), História de Portugal, Vol. VIII, Lisboa, Editorial Estampa.

FIGUEIRA, J. (2007): Os jornais como atores políticos. O Diário de Notícias, Expresso e Jornal Novo no Verão Quente de 1975, Coimbra, MinervaCoimbra.

FIGUEIRA, J. (2012): O essencial sobre a imprensa portuguesa: 1974-2010, Coimbra, Angelus Novus.

FONSECA, W. y CARVALHO, M. (2016): Heróis anónimos. Jornalismo de agência, história da ANOP e da NP 1975 -1986. Lisboa: Perfil Criativo.

GARCIA, J. L. (Org.) (2009): Estudos sobre os Jornalistas Portugueses. Metamorfoses e encruzilhadas no limiar do século XXI. Lisboa: ICS.

GASPAR, C. (2010): "O Partido Comunista e a revolução portuguesa" en Oliveira, P. A e Rezola, M. I. (Coord.), O Longo Curso. Estudos em Homenagem a José Medeiros Ferreira, Lisboa, Tinta-da-China, pp. 539-574. 
GASPAR, F. (2017): Entrevista dada a Pedro Marques Gomes. Lisboa.

GOMES, J. (2017): Entrevista dada a Pedro Marques Gomes. Lisboa.

GOMES, P. M. (2014): Os Saneamentos Políticos no Diário de Notícias no Verão Quente de 1975, Lisboa, Alêtheia Editores.

GOMES, P. M. (2018): Jornais, Jornalistas e Poder: a Imprensa que nasce na revolução e as lutas políticas de 1975. Lisboa, FCSH-NOVA (Tese de Doutoramento em História Contemporânea).

HORTA, M. T. (2011): Entrevista dada a Pedro Marques Gomes. Lisboa.

LETRIA, J. J. (2013): E tudo era possível. Retrato de juventude com Abril em fundo, Lisboa, Clube do autor.

LIMA, H. (2008): Os Diários Portuenses e os Desafios da Atualidade na Imprensa: Tradição e Ruturas. Porto, Faculdade de Letras a Universidade do Porto (Tese de Doutoramento em História).

LOURENÇO, V. (2012): Entrevista dada a Pedro Marques Gomes. Lisboa.

MATOS, A. C. (2016): “Diário de Lisboa” en Reis, a., Rezola, M. I. y Santos, P. B. (coord.), Dicionário de História de Portugal - o 25 de Abril, Vol. 3, Porto, Figueirinhas, pp. 139-140.

MAXWELL, K. (1983): The Press and the Rebirth of Iberian Democracy, Connecticut, Greenwood Press.

MESQUITA, M. (1988): “Estratégias liberais e dirigistas na comunicação social de 19741975 da comissão ad hoc à Lei de Imprensa" en Revista de Comunicação $e$ Linguagens, no 8, pp. 85-113.

MESQUITA, M. (1994a): “O Caso República: um incidente crítico" en Revista de História das Ideias, Vol. 16, pp. 507-554.

MESQUITA, M. (1994b): "Os Meios de Comunicação Social” en Reis, A. (coord.), Portugal 20 Anos de Democracia, Lisboa, Círculo de Leitores, pp. 361-405.

MESQUITA, M. (2019): "O corte revolucionário nos media e o "efeito de atraso" nas teorias da comunicação" en Media \& Jornalismo, no 35, Vol. 19, № 2, Lisboa, ICNOVA, pp. 15-22.

PALLA, M. A. (2004): "A Liberdade de Imprensa após o 25 de Abril" en Matos, A. C. y Franco, G. (org.), O Jornalismo Português: Passado, Presidente e Futuro, Lisboa, CML/HML, pp. 22-27. 
PALLA, M. A. (2013): Entrevista dada a Pedro Marques Gomes. Lisboa.

PAULO, H. (2014): “'E depois do Adeus'.. Os saneamentos e a nova legislação de censura e imprensa após o 25 de Abril en Storia $e$ Problemi Contemporanei, no 67, pp. 101-115

PINTO, A. C. (2015): “A vida política” en Pinto, A. C. (coord.), A Busca da Democracia, Lisboa, Objectiva, pp. 25-54.

RAMOS, A. R. (1994): “A Primeira de todas as liberdades" en Mesquita, M. y Rebelo, J. (Org.), O 25 de Abril nos Media Internacionais, Porto, Afrontamento, pp. 287-288.

RELATÓRIO DO CONSELHO DE IMPRENSA (1979): A Imprensa escrita em Portugal (abril de 1974 a julho de 1975). Lisboa: Conselho de Imprensa.

REIS, A. (1994): “O Processo de Democratização" en Reis, A. (coord.), Portugal 20 anos de democracia, Lisboa, Círculo de Leitores, pp. 19-39.

REZOLA, M. I. (2007): 25 de Abril: Mitos de uma Revolução, Lisboa, Esfera dos Livros.

REZOLA, M. I. (2014): “A RTP no PREC (1974-1975: Sem recuos nem Tibiezas em ordem à Limpeza Radical” en Rezola, M. I y Gomes, P. M. (Coord.). A Revolução nos Media, Lisboa, Tinta-da-China, pp. 17-51.

REZOLA, M. I. (2019): “Romper com o passado: a Revolução nos Média (Portugal, 19741975)" en Media \& Jornalismo, no 35, Vol. 19, № 2, Lisboa, ICNOVA, pp. 249-262.

RIBEIRO, N. (2002): A Rádio Renascença e o 25 de Abril, Lisboa, Universidade Católica.

RICARDO, D. (2011): Entrevista dada a Pedro Marques Gomes. Lisboa.

SANTOS, J. A. (2012): Entrevista dada a Pedro Marques Gomes. Lisboa.

SANTOS, R. (2017): A Emissora Nacional e as mudanças políticas (1968-1975), Coimbra, MinvervaCoimbra.

SASPORTES, J. (2017): Entrevista dada a Pedro Marques Gomes. Lisboa.

SEATON, J. y PIMLOTT, B. (1983): "The Portuguese media in transition" en Maxwell, K. (dir.), The Press and the Rebirth of Iberian Democracy, Connecticut, Greenwood Press, pp. 93-115.

SILVEIRA, J. (2011): A Construção do Sistema Informativo em Portugal no Séc. XX, Lisboa, Colibri.

SOUSA, P. D. (2007): A dramatização da imprensa do PREC, Coimbra, MinervaCoimbra. 
TELO, A. J. (2007): História Contemporânea de Portugal. Do 25 de Abril à atualidade, Vol. I., Lisboa, Editorial Presença.

VASCONCELOS, J. C. (2012): Entrevista dada a Pedro Marques Gomes. Lisboa. 